\title{
NADPH-dependent Secondary Amine Organocatalysis hosted by a Nucleotide-binding Domain
}

\section{Thomas Williams}

Cardiff University

\section{Yu-Hsuan Tsai}

Cardiff University https://orcid.org/0000-0003-0589-5088

Louis Luk ( $\square$ LukLY@cardiff.ac.uk)

Cardiff University

\section{Article}

Keywords: organocatalysis, NADPH-dependent hybrid catalyst

Posted Date: May 5th, 2021

DOI: https://doi.org/10.21203/rs.3.rs-468406/v1

License: (c) (i) This work is licensed under a Creative Commons Attribution 4.0 International License.

Read Full License 


\section{Abstract}

The concept of organocatalysis has been applied to facilitate "new-to-nature" reaction modes via artificial enzyme design. However, it remains challenging to recruit structurally complex natural molecules as synthetic reagents. Here, we have reported a generic design strategy that allows generation of a NADPHdependent hybrid catalyst whose action is orchestrated by a secondary amine; this system recruits a reaction mode not commonly seen among enzymes, whilst involving an intricate cofactor that cannot be used by existing organocatalysts. A secondary amine organocatalytic motif was incorporated into protein scaffolds as an unnatural amino acid by expansion of the genetic code. When introduced into the multidrug binding protein LmrR, a hybrid catalyst accepting a, $\beta$-unsaturated carbonyl substrates for transfer hydrogenation was established but was confined to the much-simplified biomimetic benzyl dihydronicotinamide (BNAH). Conversely, dihydrofolate reductase (DHFR) contains a nucleotide binding domain and can be converted into a hybrid catalyst that favourably uses NADPH for reaction, thus highlighting the importance of choosing an appropriate scaffold. The DHFR-hosted system tolerates a range of aldehyde substrates and can be coupled with an enzymatic NADPH regeneration scheme. The presented engineering approach can be readily extended to other protein scaffolds for use of different natural molecules in non-natural reaction modes.

\section{Introduction}

Organocatalysis has tremendous potentials for its applications in artificial enzyme design ${ }^{1-4}$. By hosting small organocatalytic motifs proteins can catalyse reactions with "new-to-nature" mechanisms, and various methods have been reported to generate protein-based organocatalysts ${ }^{5-15}$. However, a generic approach that recruits natural molecules as reagents is yet to be established. NAD (P)H, ATP, SAM, peptides and nucleic acids are some of the structurally complex, natural molecules that can be serve as substrates. Their use in artificial enzyme reactions will facilitate modifications of biomolecules via nonnatural reaction mechanisms and development of combined catalytic cascades, both of which underpins critical chemical and synthetic biology applications. Given the significant potentials, we have reported a design strategy that facilitates the creation of a hybrid catalyst which recruits an intricate natural cofactor NADPH in a non-natural reaction mode.

To the best of our knowledge there is currently no organocatalyst including secondary amines capable of recruiting NADPH as a synthetic reagent, though more reactive biomimetics such as benzyl dihydronicotinamide (BNAH) and Hanzstch ester have been employed as surrogates ${ }^{16-19}$. Contrarily, there are many natural enzymes utilizing NADPH for reactions, but none of them uses a secondary amine for substrate activation. Old yellow enzymes (OYE), a group of commonly used biocatalysts, activate substrates by non-covalent interactions and subsequently use flavin derivatives as a medium for hydride transfer ${ }^{20,21}$, whereas NADPH-dependent ene-reductases which utilise metal ions for substrate activation have also been reported ${ }^{22}$. Artificial metalloenzymes that use reactive transition metals to transfer hydride from $\mathrm{NAD}(\mathrm{P}) \mathrm{H}$ have also been developed ${ }^{23,24}$. Nevertheless, an NADPH-dependent catalyst that 
activates substrate by a secondary amine is needed, because its availability will help fill in the gap between the reaction repertories of bio- and organocatalysis. For instance, carbonyl substrates can be selectively activated by secondary amines within protein scaffolds through iminium or enamine catalysis, which can be further segregated into single- or two-electrons transfer ${ }^{25-27}$. NADPH, being readily recyclable through well-established regeneration schemes ${ }^{28-30}$, can be applied in organocatalytic transformations including transfer hydrogenation, dehalogenation ${ }^{31}$ and deacetoxylation ${ }^{32}$. Hence, an artificial enzyme design strategy that facilitates NADPH-dependent secondary amine organocatalysis needs to be developed, as such a catalytic system does not exist in nature.

We envisioned that an NADPH-dependent organocatalytic system can be generated by incorporating a secondary amine motif into an appropriate nucleotide binding domain. Hence, in this work unnatural amino acids bearing a cyclic secondary amine were site-specifically introduced into protein scaffolds by applying the technique of genetic code expansion ${ }^{33}$ (Fig. 1). Both the multidrug-binding protein LmrR and dihydrofolate reductase (DHFR) were tested as templates and transformed into substrate-promiscuous hybrid catalysts for reduction of various $a, \beta$-unsaturated aldehydes. Whereas the LmrR-hosted organocatalytic system is confined to BNAH as the hydride donor, the DHFR counterpart that contains a nucleotide binding domain favourably uses the natural cofactor NADPH for transfer hydrogenation. Mechanistic analysis revealed that the DHFR scaffold facilitates non-rate-limiting delivery of the pro- $R$ hydride to the iminium intermediate. To demonstrate the potential applications, the DHFR-hosted organocatalytic system was coupled to an enzymatic NADPH regeneration scheme.

\section{Results}

Genetic code expansion. Unnatural amino acids (Fig. 1a) containing a secondary amine were tested for incorporation and screened for organocatalytic activity. Amino acids 1, 2, and $\mathbf{3}$ contain D-proline, Lproline and L-thiazolidine-4-carboxylic acid (thioproline), respectively. Based on previous reports, $\mathbf{1}$ and $\mathbf{3}$ are substrates of the wild-type Methanosarcina bakeri pyrrolysyl-tRNA synthetase (MbPyIRS) and its engineered variant ThzKRS, respectively ${ }^{34,35}$. Due to the structural similarity between $\mathbf{2}$ and $\mathbf{3}$, we envisaged that 2 could be a substrate of ThzKRS. Incorporation of unnatural amino acids was tested using the reporter protein sfGFP that bears a TAG codon for the 150th amino acid residue, and formation of the full-length sfGFP was confirmed by SDS-PAGE analysis (Fig. S1). Subsequently, unnatural amino acids were incorporated into the multidrug binding protein $\mathrm{LmrR}^{36}$. Neatly presented in previous artificial enzyme design ${ }^{37-39}$, LmrR becomes catalytically competent after modifying its hydrophobic pocket, including the insertion of an unnatural amino acid. We therefore selected four previously reported residues, Val15, Asn19, Met89 and Phe93 (Fig. 1d), located in the hydrophobic pocket for substitution with 1, 2 or 3. SDS-PAGE analysis revealed successful recombinant production of the $12 \mathrm{LmrR}$ variants in E. coli (Fig. S2). The dimeric nature of these variants was confirmed by size exclusion chromatography, with the exception of LmrR-Met89-2 whose oligomeric nature appears to be hampered by the modification (see SI, Pg S7-8). Furthermore, liquid chromatography-mass spectrometry (LC-MS) investigation indicated that variants containing amino acid $\mathbf{2}$ were unstable, and up to $35 \%$ hydrolysis of 
the L-prolyl group was observed (Fig. S4). This is likely due to the presence of proline iminopeptidase in $E$. coli which cleaves $\mathrm{N}$-terminal proline in polypeptides. Such a truncation was particularly profound when $\mathbf{2}$ was at the position 89. In contrast, no detectable hydrolysis was observed in proteins carrying either Dproline 1 or L-thioproline 3 .

Use of LmrR as the scaffold for transfer hydrogenation. The LmrR variants that possess a cyclic secondary amine were then tested for their ability to mediate transfer hydrogenation (Fig. 2a). The conversion of cinnamaldehyde $\mathbf{4}$ ( 1 eq.) to its reduced counterpart $\mathbf{5}$ served as the model reaction and tested with different hydride donors. BNAH was first used, and the wild-type LmrR which contains seven lysine residues and a $\mathrm{N}$-terminal amine yielded less than $3 \%$ conversion as detected by gas chromatography-mass spectrometry (GC-MS) analysis (Fig. 2b), indicating minimal catalytic activity. Substitution of Met89 with 1, 2 or $\mathbf{3}$ showed marginal improvement ( $5 \%$ conversion $)^{40}$. Low conversion $(<15 \%)$ was observed in variants incorporated with 2 , likely due to the hydrolysis of the isopeptide bond. Substitution of thioproline 3 at the position 19 and D-proline 1 at the position 15 resulted in a detectable amount of product conversion (> 20\%). Notably, the replacement of Phe93 with $\mathbf{1}$ (LmrR-Phe93-1) was most promising giving $58 \%$ yield which is at least 19 -fold higher than that of the wild-type enzyme. Kinetic analysis of LmrR-Phe93-1 displayed enzyme-like behaviour, showing a saturation curve between reaction rate constants and BNAH (Fig. 2c) with a catalytic efficiency $k_{\text {cat }} / K_{\mathrm{M}}$ of $0.86 \mathrm{M}^{-1} \cdot \mathrm{s}^{-1}$.

When NADPH was tested as the hydride source for organocatalysis by LmrR-Phe93-1, no conversion was detected. Whilst disappointing, this observation aligns with the literatures. NADH was found to be a poor hydride donor for small organocatalytic secondary amines, and organic solvents was used for these reactions ${ }^{16,41}$. Whereas $\mathbf{1 - 3}$ are water soluble, transfer hydrogenation with NADPH by these secondary amines in aqueous buffer was not observed. This is also the case for the protein-hosted secondary amines presented here and previously ${ }^{11}$. It is likely that the dinucleotide hinders the dihydronicotinamide motif from approaching the intermediate for reactions. Hence, a protein scaffold that can place the reacting centres in proximity is desired.

Use of DHFR as the scaffold for transfer hydrogenation. Seeking an NAPDH-dependent organocatalytic system, we extended to examine a template that contains a domain naturally evolved for binding nucleotide-containing cofactors ${ }^{42}$. Escherichia coli dihydrofolate reductase (DHFR) is a well-investigated enzyme that catalyses the step of hydride transfer from the C4 pro- $R$ hydride of NADPH to $\mathrm{C} 6$ of dihydrofolate with concomitant protonation at the N5 position in the one-carbon metabolism for thymidine biosynthesis ${ }^{43}$. DHFR contains a Rossmann fold, which is composed of a multi-stranded $\beta$ sheet packed by two a helices on each side and binds nucleotide-containing cofactor NADPH with an association rate up to $10^{5} \mathrm{M}^{-1} \cdot \mathrm{s}^{-144,45}$. Additionally, it contains a mobile M20 loop (residue 9-23) which closes the active site upon binding NADPH generating a shielded environment surrounding that nicotinamide motif ${ }^{46,47}$. These features present DHFR as an ideal scaffold for transfer hydrogenation by NADPH through introduction of a cyclic secondary amine organocatalyst. To this end, Ala7, Phe31 and Ser49 that are in proximity to the nicotinamide motif of the NADPH binding site were individually replaced 
with 1 (Fig. 1e). The resulting DHFR variants were verified by SDS-PAGE (Fig. S3) and mass spectrometry (Fig. S5).

Activity tests revealed that DHFR variants incorporated with $\mathbf{1}$ could facilitate NADPH-dependent transfer hydrogenation (Fig. 2a). For the wild-type DHFR, the reaction product 5 was undetectable by GC-MS analysis. Replacement of Ser49 with 1 led to considerable protein precipitation with no detectable products. Contrarily, replacement of Ala7 with 1 could effectively catalyse the reaction as evident by significant product conversion (Fig. 2d). Though BNAH can be used as the hydride source, the extent of product conversion was noticeably higher when utilising NADPH. With 0.1 equivalent ( $10 \mathrm{~mol} \%$ ) of DHFRAla7-1, 90\% of starting material $\mathbf{4}$ was converted into product $\mathbf{5}$ in aqueous buffer at pH 7.0 using 2 equivalents of NADPH after 18 hours (versus $20 \%$ conversion with BNAH under the same conditions). DHFR-Ala7-1 also demonstrated enzyme-like kinetic behaviour showing a saturation curve between the rate constants and cofactor concentration with catalytic efficiency $\left(k_{\text {cat }} / K_{\mathrm{M}}\right)$ reaching $5.77 \mathrm{M}^{-1} \cdot \mathrm{s}^{-1}$ (Fig. 2e). It is noteworthy that, while DHFR-Ala7-1 uses the structurally complex NADPH for reaction, its catalytic efficiency $\left(k_{\text {cat }} / K_{\mathrm{M}}\right)$ is $\sim$ 5-fold higher than that of LmrR-Phe93-1 measured with BNAH.

Reaction modes used by LmrR-Phe93-1 and DHFR-Ala7-1. Similar to many other secondary amine organocatalysts ${ }^{48}$, formation of iminium ion is critical towards carbonyl substrate activation, and thus its transient existence during the catalysis by LmrR-Phe93-1 and DHFR-Ala7-1 was examined for mechanistic insights. The two variants were incubated with the starting material 4, treated with $\mathrm{NaCNBH}_{3}$ and subjected to high resolution mass spectrometric analysis, as previously described (see $\mathrm{SI})^{3}$. The LmrR-Phe93-1 sample predominantly yielded one protein species with an observed mass matching the calculated molecular weight of the reduced covalent protein-substrate intermediate (Fig. S6A). Similarly, a substantial portion of the DHFR-Ala7-1 variant also yielded the reduced covalent intermediate under the same condition, but hydrolysis of the prolyl-group was also observed upon treatment with $\mathrm{NaCNBH}_{3}(\mathrm{Fig}$. S6B). Treatment of these protein species with chymotrypsin revealed digested peptides, whose elemental composition identified by liquid chromatography tandem mass spectrometry (LC-MS/MS) within $1 \mathrm{ppm}$ accuracy corresponds to the trapped intermediate (Fig. S7 and S8). In contrast, the wild-type LmrR and DHFR that cannot catalyse the transfer hydrogenation did not show any evidence of the iminium intermediate formation. Accordingly, these results support a non-natural LUMO-lowering reaction mechanism ${ }^{48}$, in which the secondary amine activates the starting material through iminium ion formation for transfer hydrogenation from the corresponding hydride donor (Fig. 3).

Contrary to previous work which relied on a LmrR-hosted aniline for catalysis ${ }^{3}$, no lysine modification was detected in our protein scaffolds after $\mathrm{NaCNBH}_{3}$ treatment. The chemoselectivity observed in this work implies the significant reactivity of the secondary amine, enabling iminium intermediate formation with relatively inert carbonyl substrate under aqueous conditions. Furthermore, in LmrR the aniline organocatalysis was found to be optimal at position $15^{3}$, whereas we obtained higher conversions with the secondary amine organocatalyst at position 93 , indicating that activity test at different positions is essential during protein-based catalyst design. 
Prochirality and kinetic insights of the transfer hydrogenation reaction catalysed by DHFR-Ala7-1. When [4 $\left.R^{-2} \mathrm{H}\right]-\mathrm{NADPH}$ (NADPD) was used in the catalysis by DHFR-Ala7-1, only one product isotopologue with the deuteride located at the $\mathrm{C}_{\beta}$ position was identified by GC-MS (Fig. S9). This observation agrees with previous crystal and biochemical analysis which illustrated that the wild-type DHFR catalyses the transfer of the $\mathrm{C} 4$ pro- $R$ hydride of $\mathrm{NADPH}^{49,50}$. Furthermore, this protein-hosted organocatalyst is regioselective favouring 1,4- over 1,2-addition, the latter was observed in the streptavidin-hosted secondary amine organocatalytic system as a side reaction ${ }^{10}$. Given its stereoselectivity, primary kinetic isotope effect (KIE) of hydride transfer was assessed. By comparing the turnover rate constants under saturating conditions between the reactions that use NADPH and NADPD, a kinetic isotope effect (KIE) of $1.10 \pm 0.16$ (Fig. S10) was obtained, implying that the step of hydride transfer is not rate-limiting. Other chemical step(s) such as iminium ion formation can be rate-limiting in this multi-step catalytic cycle ${ }^{51}$. Alternatively, the physical step of releasing the oxidised cofactor from the enzyme can dictate the rate of catalytic turnover, as observed in the natural reaction catalysed by the wild-type enzyme ${ }^{44,47}$.

Substrate Scope. With the active systems in hand, substrate promiscuity of LmrR-Phe93-1 and DHFRAla7-1 were investigated. Aromatic and ketone analogues of cinnamaldehyde were evaluated as alternative substrates. Both systems were able to accept all the aldehydes tested for the selective reduction. With respect to per mol\% of catalyst, the conversions of aldehydes to the corresponding reduced products by DHFR-Ala7-1 was generally higher than those by LmrR-Phe93-1 (Fig. 4, Table S6 and S7). On the other hand, the ketone substrate showed no turnover in either system. Hence, two proteinbased organocatalytic systems, with one preferentially using BNAH and the other NADPH, that have substrate promiscuity for aldehydes have been developed.

Coupling of the NADPH-dependent organocatalysis with an enzymatic regeneration scheme. Although it is structurally complex, NADPH is commonly used in biocatalysis for chemical manufacturing through coupling of cofactor regeneration schemes which excel at their specificity, efficiency and ease of handling ${ }^{30}$. Hence, we coupled the organocatalysis by DHFR-Ala7-1 with an enzymatic regeneration scheme enabling sub-stoichiometric use of NADPH (Fig. 5a). Importantly, this experiment proves the concept that secondary amine organocatalysis, amongst other chemical catalytic systems ${ }^{52}$, can be driven by a well-established biocatalysis tool. The conversion of starting material $\mathbf{4}$ to product $\mathbf{5}$ by DHFRAla7-1 was performed in the presence of the reaction by glucose 6-phosphate dehydrogenase (G6PDH), a commercially available enzyme that oxidises glucose 6-phosphate whilst reducing $\mathrm{NADP}^{+}$to regenerate $\mathrm{NADPH}^{29}$. The coupling between the organocatalytic and enzymatic reactions was assessed by the product conversion and the total turnover number, which is defined as the number of moles of product formed per mole of cofactor used (Table S8) ${ }^{29}$. The product conversion could reach up to $90 \%$ when the coupling reaction was included, whereas under the same reaction conditions the conversion was barely detectable ( $<1 \%$ ) in the absence of G6PDH (Fig. 5b). Furthermore, the total turnover number was measured up to 10460 reaching the detection limit of our experimental approach (Fig. 5c). A value of this magnitude is arguably economically viable on an industrial scale $28,30,53$, highlighting the significant potential of the presented NADPH-dependent organocatalytic system for synthetic applications. Such 
work complements with the recent progress made in using alternative hydride donors in enzymatic reactions ${ }^{54}$, and together they will eventually enhance the merger of chemical and biocatalysis.

\section{Conclusion}

A NADPH-dependent secondary amine organocatalytic system enabled by use of a nucleotide binding domain has been established. Notably, NADPH can be now used as a synthetic reagent by an organocatalytic motif, whereas aldehyde substrates activated by a secondary amine for transfer hydrogenation is a "new-to-nature" enzyme reaction mode. Though the same substrate activation mode was used between the LmrR and DHFR variants, only the latter can use the natural cofactor for reactions, underlining the importance of the protein scaffold and the design strategy. Furthermore, this work complements with previous reports, where $p$-aminophenylalanine and $\mathrm{N}$-methyl histidine were introduced into protein scaffolds for organocatalytic transformations $s^{3,4,55,56}$. In both of these cases a protein scaffold was chosen and then optimised for reactions involving relatively small reagents ( $200 \mathrm{Da})$, while in this work a sizeable structurally complex natural metabolite was used and laboratory evolution has yet to be applied. Lastly, our protein engineering strategy is most likely transferrable, facilitating the development of non-natural reaction modes that recruit natural metabolites as reagents.

\section{Methods}

All methods reagents and characterisation can be found in the supplementary material.

\section{Declarations}

\section{Affiliation:}

*School of Chemistry

Cardiff University

Main Building, Park Place

Cardiff, CF10 3AT

UK

${ }^{\#}$ Cardiff Catalysis Institute,

School of Chemistry,

Cardiff University,

Main Building, Park Place, 


\section{Contributions:}

T.L.W. performed the experimental work. All authors designed and planned the work. Initial draft was written by L.Y.P.L. All authors wrote the final article. All authors have given approval to the final version of the manuscript.

\section{Acknowledgement:}

We would like to thank EPSRC (EP/L027240/1) for the HR-LCMS resources and Cardiff School of Chemistry for funding Thomas Williams' PhD. We would like to thank for the financial support provided by BBSRC (BB/T015799/1), Leverhulme Trust (RPG-2017-195), Royal Society (RG170187) and Wellcome Trust (202056/Z/16/Z) for Louis Y. P. Luk. We would like to thank Professor Dr Jason Chin at the Medical Research Council Laboratory of Molecular Biology (MRC-LMB) for MbPyIRS plasmid and Professor Dr Rudolf $\mathrm{K}$ Allemann for the DHFR and tbADH plasmids.

\section{References}

1 Groger, H. \& Hummel, W. Combining the 'two worlds' of chemocatalysis and biocatalysis towards multi-step one-pot processes in aqueous media. Curr. Opin. Chem. Biol. 19, 171-179, (2014).

2 van der Helm, M. P., Klemm, B. \& Eelkema, R. Organocatalysis in aqueous media. Nat. Rev. Chem. 3, 491-508, (2019).

3 Drienovska, I., Mayer, C., Dulson, C. \& Roelfes, G. A designer enzyme for hydrazone and oxime formation featuring an unnatural catalytic aniline residue. Nat. Chem. 10, 946-952, (2018).

4 Burke, A. J. et al. Design and evolution of an enzyme with a non-canonical organocatalytic mechanism. Nature 570, 219-223, (2019).

5 Levine, H. L., Nakagawa, Y. \& Kaiser, E. T. Flavopapain: synthesis and properties of semi-synthetic enzymes. Biochem. Biophys. Res. Commun. 76, 64-70, (1977).

6 Zandvoort, E., Geertsema, E. M., Quax, W. J. \& Poelarends, G. J. Enhancement of the promiscuous aldolase and dehydration activities of 4-oxalocrotonate tautomerase by protein engineering.

ChemBioChem 13, 1274-1277, (2012).

7 Guo, C. et al. Biocatalytic Asymmetric Michael Additions of Nitromethane to alpha,betaUnsaturated Aldehydes via Enzyme-bound Iminium Ion Intermediates. ACS Catal. 9, 4369-4373, (2019).

8 Biewenga, L. et al. Enantioselective Synthesis of Pharmaceutically Active gamma-Aminobutyric Acids Using a Tailor-Made Artificial Michaelase in One-Pot Cascade Reactions. ACS Catal. 9, 1503-1513, (2019). 
9 Nodling, A. R. et al. Reactivity and Selectivity of Iminium Organocatalysis Improved by a Protein Host. Angew. Chem. Int. Ed. 57, 12478-12482, (2018).

10 Santi, N., Morrill, L. C. \& Luk, L. Y. P. Streptavidin-Hosted Organocatalytic Aldol Addition. Molecules 25, 2457, (2020).

11 Santi, N. et al. Transfer hydrogenations catalyzed by streptavidin-hosted secondary amine organocatalysts. Chem. Commun. 57, 1919-1922, (2021).

12 Jiang, L. et al. De novo computational design of retro-aldol enzymes. Science 319, 1387-1391, (2008).

13 Althoff, E. A. et al. Robust design and optimization of retroaldol enzymes. Protein Sci. 21, 717-726, (2012).

14 Obexer, R. et al. Emergence of a catalytic tetrad during evolution of a highly active artificial aldolase. Nat. Chem. 9, 50-56, (2017).

15 Cotelle, Y. et al. Anion-pi Enzymes. ACS Cent. Sci. 2, 388-393, (2016).

16 Brogan, A. P., Dickerson, T. J. \& Janda, K. D. Nornicotine-organocatalyzed aqueous reduction of alpha,beta-unsaturated aldehydes. Chem. Commun., 4952-4954, (2007).

17 Löw, S. A., Löw, I. M., Weissenborn, M. J. \& Hauer, B. Enhanced Ene-Reductase Activity through Alteration of Artificial Nicotinamide Cofactor Substituents. ChemCatChem 8, 911-915, (2016).

18 Faisca Phillips, A. M. \& Pombeiro, A. J. Recent advances in organocatalytic enantioselective transfer hydrogenation. Org. Biomol. Chem. 15, 2307-2340, (2017).

19 Mathew, S. et al. Enantio- and regioselective ene-reductions using $\mathrm{F} 420 \mathrm{H} 2$-dependent enzymes. Chem. Commun. 54, 11208-11211, (2018).

20 Peters, C., Frasson, D., Sievers, M. \& Buller, R. Novel Old Yellow Enzyme Subclasses. ChemBioChem 20, 1569-1577, (2019).

21 Toogood, H. S., Gardiner, J. M. \& Scrutton, N. S. Biocatalytic Reductions and Chemical Versatility of the Old Yellow Enzyme Family of Flavoprotein Oxidoreductases. Chemcatchem 2, 892-914, (2010).

22 Mansell, D. J. et al. Biocatalytic Asymmetric Alkene Reduction: Crystal Structure and Characterization of a Double Bond Reductase from Nicotiana tabacum. ACS Catal. 3, 370-379, (2013).

23 Okamoto, Y., Köhler, V. \& Ward, T. R. An NAD(P)H-Dependent Artificial Transfer Hydrogenase for Multienzymatic Cascades. J. Am. Chem. Soc. 138, 5781-5784, (2016). 
24 Okamoto, Y. \& Ward, T. R. Transfer Hydrogenation Catalyzed by Organometallic Complexes Using NADH as a Reductant in a Biochemical Context. Biochemistry 56, 5223-5224, (2017).

25 Nicewicz, D. A. \& MacMillan, D. W. Merging photoredox catalysis with organocatalysis: the direct asymmetric alkylation of aldehydes. Science 322, 77-80, (2008).

26 Schweitzer-Chaput, B., Horwitz, M. A., de Pedro Beato, E. \& Melchiorre, P. Photochemical generation of radicals from alkyl electrophiles using a nucleophilic organic catalyst. Nat. Chem. 11, 129-135, (2019).

27 Murphy, J. J. et al. Asymmetric catalytic formation of quaternary carbons by iminium ion trapping of radicals. Nature 532, 218-222, (2016).

28 Chenault, H. K. \& Whitesides, G. M. Regeneration of nicotinamide cofactors for use in organic synthesis. Appl. Biochem. Biotechnol. 14, 147-197, (1987).

29 Wong, C.-H. \& Whitesides, G. M. Enzyme-catalyzed organic synthesis: NAD(P)H cofactor regeneration by using glucose-6-phosphate and the glucose-5-phosphate dehydrogenase from Leuconostoc mesenteroides. J. Am. Chem. Soc. 103, 4890-4899, (1981).

30 Chenault, H. K., Simon, E. S. \& Whitesides, G. M. Cofactor regeneration for enzyme-catalysed synthesis. Biotechnol. Genet. Eng. Rev. 6, 221-270, (1988).

31 Emmanuel, M. A., Greenberg, N. R., Oblinsky, D. G. \& Hyster, T. K. Accessing non-natural reactivity by irradiating nicotinamide-dependent enzymes with light. Nature 540, 414-417, (2016).

32 Biegasiewicz, K. F. et al. Catalytic promiscuity enabled by photoredox catalysis in nicotinamidedependent oxidoreductases. Nat. Chem. 10, 770-775, (2018).

33 Nodling, A. R. et al. Using genetically incorporated unnatural amino acids to control protein functions in mammalian cells. Essays Biochem. 63, 237-266, (2019).

34 Polycarpo, C. R. et al. Pyrrolysine analogues as substrates for pyrrolysyl-tRNA synthetase. FEBS Lett. 580, 6695-6700, (2006).

35 Nguyen, D. P. et al. Genetically encoded 1,2-aminothiols facilitate rapid and site-specific protein labeling via a bio-orthogonal cyanobenzothiazole condensation. J. Am. Chem. Soc. 133, 11418-11421, (2011).

36 Agustiandari, $\mathrm{H}$. et al. $\mathrm{LmrR}$ is a transcriptional repressor of expression of the multidrug $A B C$ transporter LmrCD in Lactococcus lactis. J. Bacteriol. 190, 759-763, (2008).

37 Roelfes, G. LmrR: A Privileged Scaffold for Artificial Metalloenzymes. Acc. Chem. Res. 52, 545-556, (2019). 
38 Bos, J., Fusetti, F., Driessen, A. J. \& Roelfes, G. Enantioselective artificial metalloenzymes by creation of a novel active site at the protein dimer interface. Angew. Chem. Int. Ed. 51, 7472-7475, (2012).

39 Drienovska, I. et al. Design of an enantioselective artificial metallo-hydratase enzyme containing an unnatural metal-binding amino acid. Chem. Sci. 8, 7228-7235, (2017).

40 Madoori, P. K., Agustiandari, H., Driessen, A. J. \& Thunnissen, A. M. Structure of the transcriptional regulator LmrR and its mechanism of multidrug recognition. EMBO J. 28, 156-166, (2009).

41 Ouellet, S. G., Tuttle, J. B. \& MacMillan, D. W. Enantioselective organocatalytic hydride reduction. J. Am. Chem. Soc. 127, 32-33, (2005).

42 Medvedev, K. E. et al. A Fifth of the Protein World: Rossmann-like Proteins as an Evolutionarily Successful Structural unit. J. Mol. Biol. 433, 166788, (2021).

43 Luk, L. Y. et al. Unraveling the role of protein dynamics in dihydrofolate reductase catalysis. Proc. Natl. Acad. Sci. U. S. A. 110, 16344-16349, (2013).

44 Fierke, C. A., Johnson, K. A. \& Benkovic, S. J. Construction and evaluation of the kinetic scheme associated with dihydrofolate reductase from Escherichia coli. Biochemistry 26, 4085-4092, (1987).

45 Zhang, Z. et al. Single-molecule and transient kinetics investigation of the interaction of dihydrofolate reductase with NADPH and dihydrofolate. Proc. Natl. Acad. Sci. U. S. A. 101, 2764-2769, (2004).

46 Miller, G. P. \& Benkovic, S. J. Strength of an interloop hydrogen bond determines the kinetic pathway in catalysis by Escherichia coli dihydrofolate reductase. Biochemistry 37, 6336-6342, (1998).

47 Luk, L. Y., Loveridge, E. J. \& Allemann, R. K. Protein motions and dynamic effects in enzyme catalysis. Phys. Chem. Chem. Phys. 17, 30817-30827, (2015).

48 Erkkila, A., Majander, I. \& Pihko, P. M. Iminium catalysis. Chem. Rev. 107, 5416-5470, (2007).

49 Sawaya, M. R. \& Kraut, J. Loop and subdomain movements in the mechanism of Escherichia coli dihydrofolate reductase: crystallographic evidence. Biochemistry 36, 586-603, (1997).

50 Stojkovic, V. et al. Effects of the donor-acceptor distance and dynamics on hydride tunneling in the dihydrofolate reductase catalyzed reaction. J. Am. Chem. Soc. 134, 1738-1745, (2012).

51 Swiderek, K. et al. Reaction Mechanism of Organocatalytic Michael Addition of Nitromethane to Cinnamaldehyde: A Case Study on Catalyst Regeneration and Solvent Effects. J. Phys. Chem. A 122, 451459, (2018).

52 Kohler, V. et al. Synthetic cascades are enabled by combining biocatalysts with artificial metalloenzymes. Nat. Chem. 5, 93-99, (2013). 
Opin. Biotechnol. 14, 421-426, (2003).

54 Guarneri, A., van Berkel, W. J. H. \& Paul, C. E. Alternative coenzymes for biocatalysis. Curr. Opin. Biotechnol. 60, 63-71, (2019).

55 Zhou, Z. \& Roelfes, G. Synergistic catalysis in an artificial enzyme by simultaneous action of two abiological catalytic sites. Nat. Catal. 3, 289-294, (2020).

56 Mayer, C. et al. Directed Evolution of a Designer Enzyme Featuring an Unnatural Catalytic Amino Acid. Angew. Chem. Int. Ed. 58, 2083-2087, (2019).

Figures
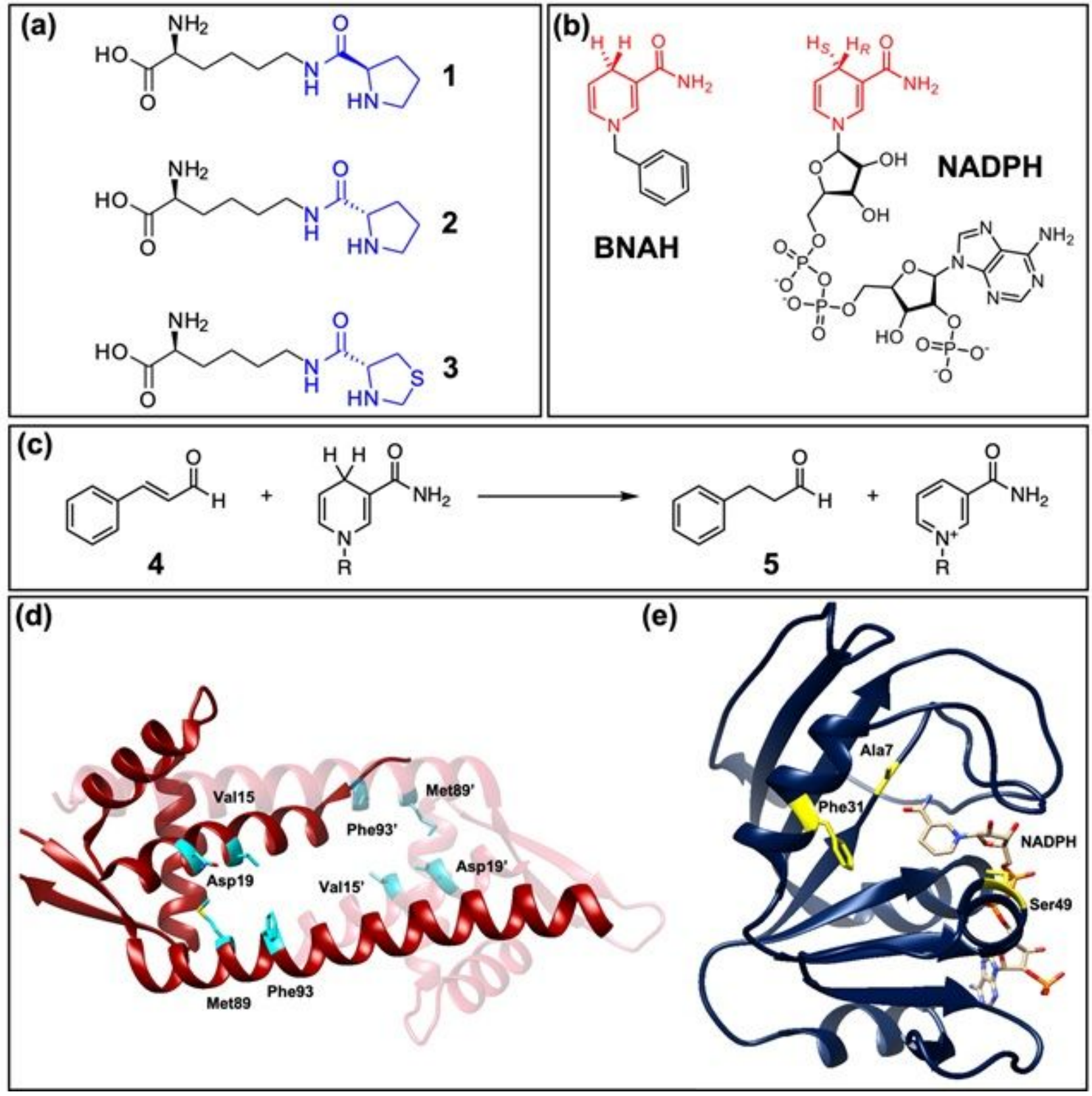
Figure 1

Components used in the design and testing of the protein-based organocatalytic system. (a) The structures of the unnatural amino acids: D-prolyl-L-lysine 1, L-prolyl-L-lysine 2 and L-thiazolidine-L-lysine 3. (b) The structures of the hydride donors, benzyl dihydronicotinamide (BNAH) and nicotinamide adenine dinucleotide phosphate (NADPH). (c) organocatalytic reduction of cinnamaldehyde 4 to the corresponding saturated hydro-cinnamaldehyde 5 by a hydride donor. (d) The X-ray crystal structure of the Lactococcus multidrug resistant regulator (LmrR) protein homodimer (PDB: 3F8F). Two monomers are shown in red and light red. Residues targeted for unnatural amino acid incorporation are highlighted in cyan. (e) The X-ray crystal structure of E. coli dihydrofolate reductase (DHFR) with NADPH bound in the active site (PDB: 1RA1). Residues targeted for unnatural amino acid incorporation are highlighted in yellow.

(a)<smiles>[R]N1C=CCC(C(N)=O)=C1</smiles>

(b)

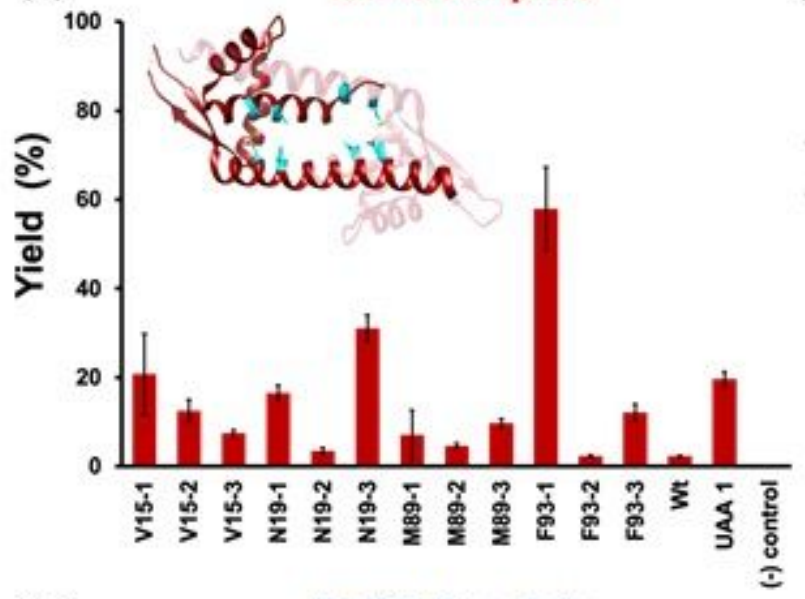

(d)

DHFR Template

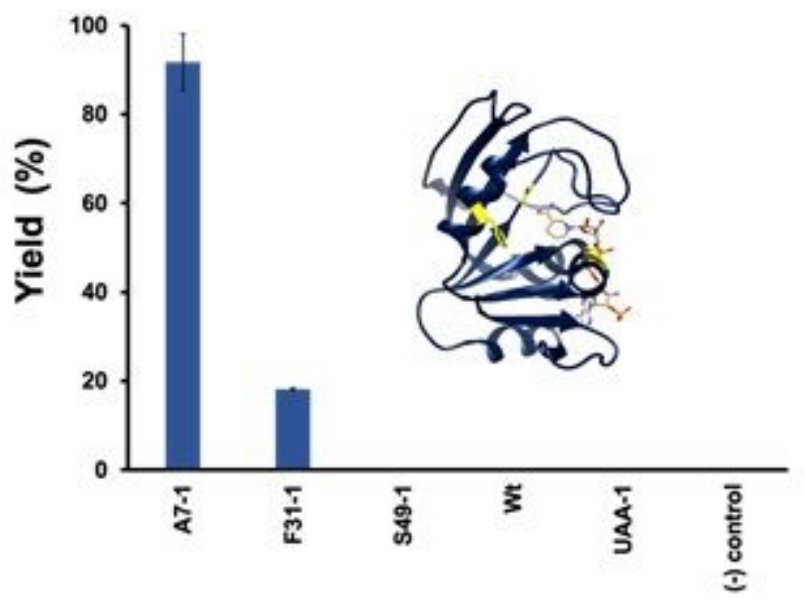

(c) LmrR-Phe93-1

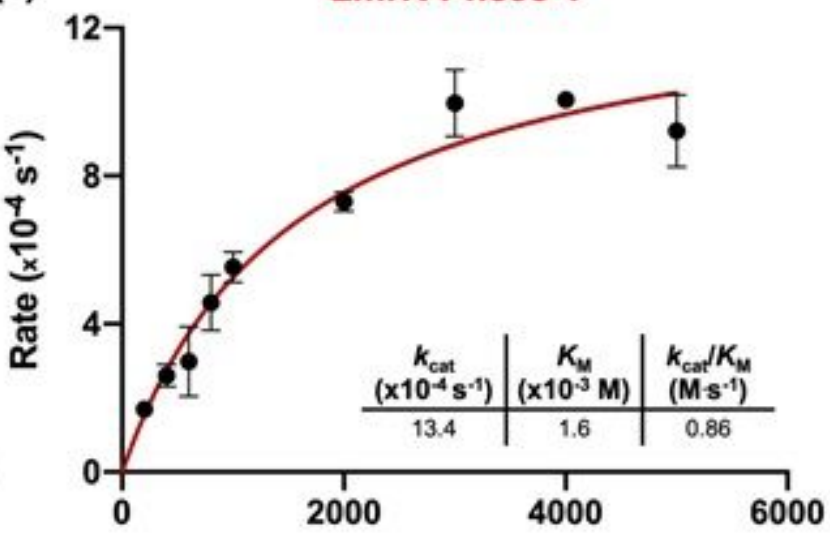

[BNAH] ( $\mu \mathrm{M})$

(e)

DHFR-Ala7-1

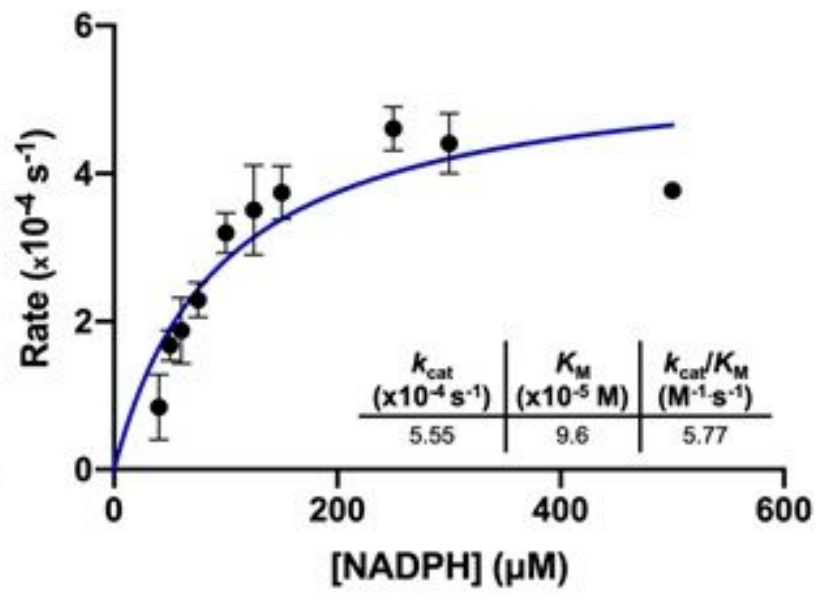

Figure 2 
Assessment of the catalytic efficiency in the transfer hydrogenation reaction. (a) The model organocatalytic transfer hydrogenation reaction using either BNAH (LmrR) or NADPH (DHFR) at $25^{\circ} \mathrm{C}$ for 18 h. $10 \%$ and $5 \%$ Methanol was used in the LmrR and DHFR reactions, respectively. (b) Yield of the model reaction catalysed by the LmrR variants using BNAH as determined by GC-MS. (c) MichaelisMenten kinetic curve determined by varying the concentration of BNAH using LmrR-Phe93-1 as the catalyst. (d) Yield of the model reaction catalysed by the DHFR variants using NADPH as determined by GC-MS. (e) Michaelis-Menten kinetic curve determined by varying the concentration of NADPH using DHFR-Ala7-1 as the catalyst. The template experiment (b) and (d) were tested alongside the wild-type proteins, 1 and a negative (-) control where the reaction was performed without any catalyst. Each reaction was performed in triplicate and the mean ( \pm standard deviation) is shown.

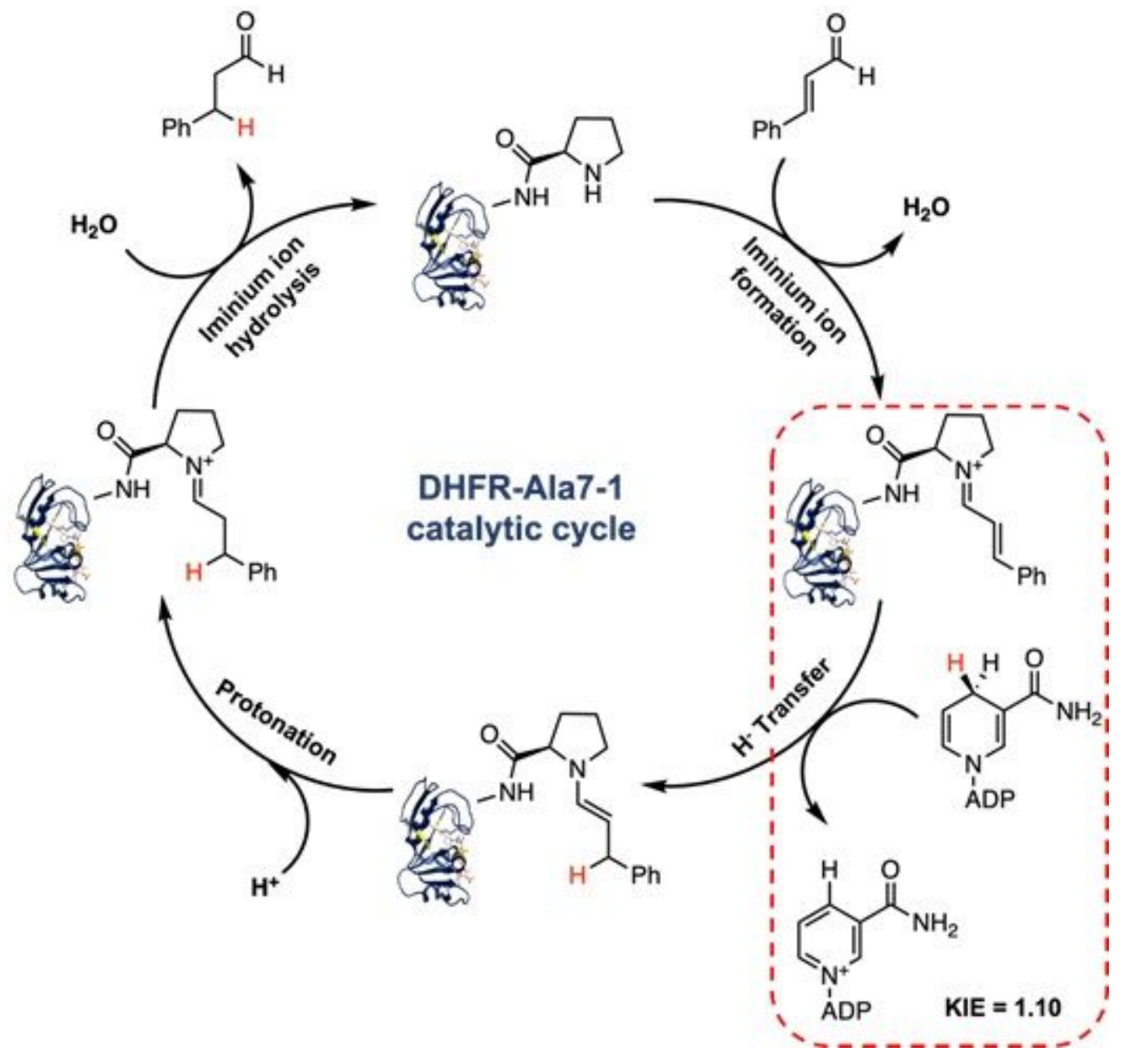

Figure 3

Proposed catalytic cycle for the organocatalytic transfer hydrogenation reaction by DHFR-Ala7-1. The unnatural amino acid forms an iminium ion with the $a, \beta$-unsaturated carbonyl substrate, and hydride transfer occurs from the pro-R position of $\mathrm{NADPH}$ to $\mathrm{C} \beta$ of the iminium intermediate. 


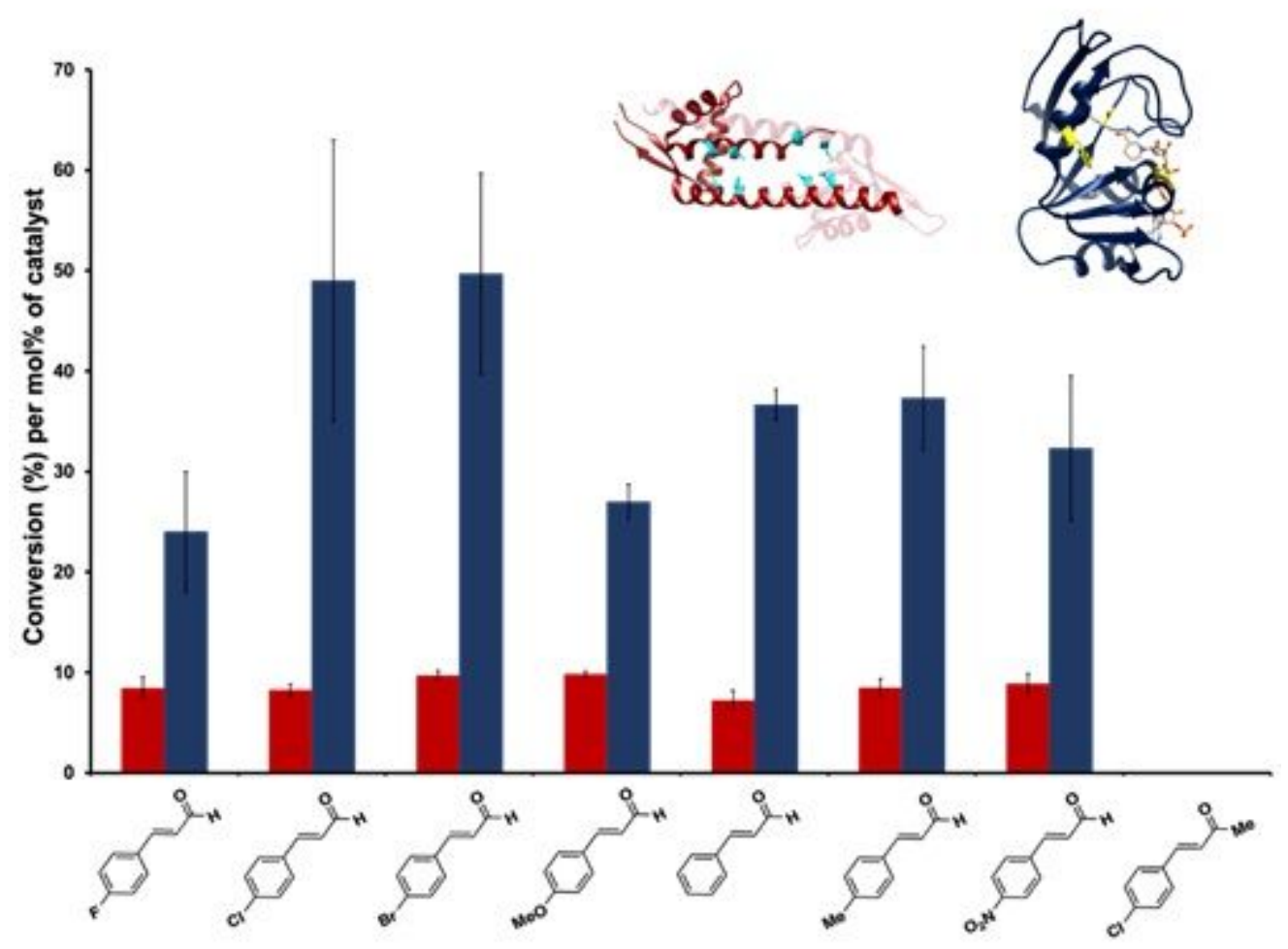

Figure 4

Substrate scope analysis of the organocatalytic proteins LmrR-Phe93-1 and DHFR-Ala7-1. The LmrR or DHFR variant was incubated with the indicated $a, \beta$-unsaturated carbonyl compound and 5 eq of their respective hydride donors (BNAH and NADPH, respectively). Conversion (\%) per mol\% was determined by comparison of the integrals of the protons arising from the aldehyde protons of the saturated substrate to the unsaturated product as revealed by $1 \mathrm{H}$ NMR spectroscopy (see SI). For the ketone, no change in the substrate could be observed. Each reaction was performed in triplicate and the mean yield ( \pm standard deviation) reported.
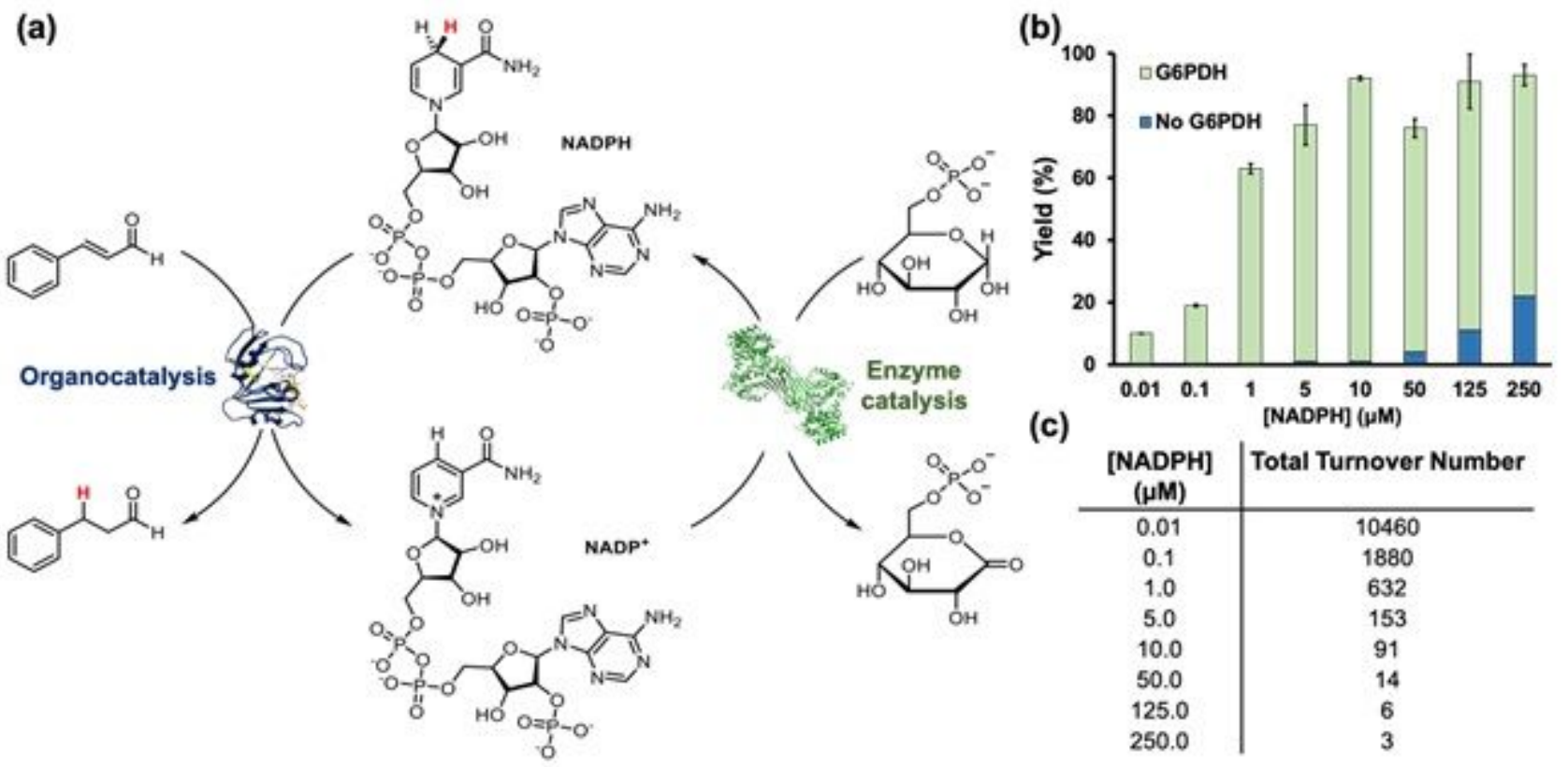


\section{Figure 5}

Reaction scheme, total turnover number and product yield of the coupling scheme between the organocatalytic DHFR-Ala7-1 and the enzymatic glucose-6-phosphate dehydrogenase (G6PDH) reactions. (a) The organocatalytic transfer hydrogenation reaction of cinnamaldehyde 4 (1 mM) by DHFR-Ala7-1 (10 mol\%) was driven by the enzymatic G6PDH reaction ( $50 \mathrm{nM}$ ) which oxidises glucose-6-phoshate (2 mM) to the corresponding lactone with the concomitant NADPH regeneration. (b) Product conversion of the organocatalytic reaction with and without the cofactor recycling system as estimated by GC ( \pm standard deviation). (c) The total turnover number observed with respect to the concentration of NADPH. Each reaction was performed in triplicate and the mean reported.

\section{Supplementary Files}

This is a list of supplementary files associated with this preprint. Click to download.

- SupplementaryInformation.docx 